\title{
Nonlinear Dynamical Modeling of Solar Cycles Using Dynamo Formulation with Turbulent Magnetic Helicity
}

\author{
I.N. Kitiashvili*1 \& A.G. Kosovichev ${ }^{2}$ \\ ${ }^{1}$ Center for Turbulence Research, Stanford University, Stanford, CA 94305, USA \\ ${ }^{2}$ W.W. Hansen Experimental Physics Laboratory, Stanford University, Stanford, CA 94305, USA
}

(Received 24 April 2008; in final form 25 July 2008)

\begin{abstract}
Variations of the sunspot number are important indicators of the solar activity cycles. The sunspot formation is a result of a dynamo process inside the Sun, which is far from being understood. We use simple dynamical models of the dynamo process to simulate the magnetic field evolution and investigate general properties of the sunspot number variations during the solar cycles. We have found that the classical Parker's model with a standard kinetic helicity quenching cannot represent the typical profiles of the solar-cycle variations of the sunspot number, and also does not give chaotic solutions. For modeling of the solar cycle properties we use a nonlinear dynamo model of Kleeorin and Ruzmaikin (1982), which takes into account dynamics of the turbulent magnetic helicity. We have obtained a series of periodic and chaotic solutions for different layers of the convective zone. The solutions qualitatively reproduce some basic observational features of the solar cycle properties, in particular, the relationship between the growth time and the cycle amplitude. Also, on the longer time scale the dynamo model with the magnetic helicity has intermittent solutions, which may be important for modeling long-term variations of the solar cycles.
\end{abstract}

\section{Introduction}

Investigation of solar activity has a long history. The 11-year sunspot cycles were discovered by Schwabe (1844). Detailed observations of sunspots locations on the solar disk and their sizes provided the latitudinal distribution during the cycle, known as the "butterfly diagram". Discovered by Hale (1919) the flip-flop phenomenon of the global solar magnetic field with a period of 22 years showed the connection between variations of the sunspots number and the magnetic field evolution. Taking into account the Hale's law Bracewell (1953) proposed to include the information about the periodical reversals of the magnetic field in the sunspot number series by assigning alternating positive and negative signs to the sunspot cycles, thus representing the sunspot cycles as a 22-year oscillation. Bracewell suggested that the nonlinear temporal distribution of the sunspots number can be written in the form of a three-halfs law (Bracewell 1988): $W(t)=100\left[\left|W_{\text {lin }}(t)\right| / 83\right]^{3 / 2}$, where $W_{\text {lin }}(t)$ is a new "linearized" sunspot number, which is supposedly proportional to a typical strength of the Sun's toroidal magnetic field.

For an explanation of the magnetic field generation Parker (1955) proposed a simple dynamo model, which describes the phenomenon as an action of two factors: the differential rotation and cyclonic convective vortices. The mean-field theory and discovery of the $\alpha$-effect give us a general description of the process of magnetic field generation (Krause and Rädler 1980). For recent reviews we refer to Charbonneau (2005) and Brandenburg and Subramanian (2005).

It is known that the dynamo process is characterized by algebraic and dynamic nonlinearities (e.g. Kleeorin and Rogachevskii 2007, Sokoloff 2007). The algebraic nonlinearity can be determined as influence of the magnetic field on fluid motions and on the kinetic helicity. This results in quenching of the electromotive force and limits the growth of the magnetic field. The evolution of the small-scale magnetic helicity in the turbulent plasma causes a dynamical nonlinearity in the dynamo process.

\footnotetext{
${ }^{*}$ Corresponding author. Email: irinasun@stanford.edu

Geophysical and Astrophysical Fluid Dynamics

ISSN 0309-1929 print/ ISSN 1029-0419 online (C)2006 Taylor \& Francis Ltd http://www.tandf.co.uk/journals DOI: $10.1080 / 03091920 \times x \times x \times x \times x x$
} 
The turbulent helicity conditionally can be divided into two parts: hydrodynamic and magnetic. The kinetic helicity describes helical turbulent fluid motions; the magnetic helicity determines the order of twisted magnetic field lines. Due to the fact that the kinetic helicity makes the magnetic field small-scaled, the back influence on the turbulent fluid motions can restrict the unlimited growth of the magnetic field. In the mean-field approach the magnetic helicity is separated into large- and small-scale components. Because of the conservation of the total helicity a growth of the large-scale magnetic helicity due to the dynamo action is compensated by the growth of the small-scale helicity of opposite sign (Sokoloff 2007). Thus, the small- and large-scale magnetic fields grow together and are mirror-asymmetrical. This means that the condition of magnetic helicity conservation is, perhaps, more severe for a restriction of the dynamo action than the condition of the energy conservation, which leads to quenching of the kinetic helicity. Since the concept of magnetic helicity was introduce into the dynamo theory by Pouquet et al. (1976) many magnetic helicity models were suggested. For modeling the solar cycle we choose the formulation of Kleeorin and Ruzmaikin (1982), explicitly based on the idea of magnetic helicity conservation. Observational data of solar magnetic fields are in a reasonable agreement with the idea of the magnetic helicity conservation (Kleeorin et al. 2003, Zhang et al. 2006, Sokoloff 2007, Sokoloff et al. 2007). Similar dynamo models with magnetic helicity have been also considered in the context of galactic dynamos (Kleeorin et al. 2000) and have included helicity transport by mean flows (Sur et al. 2007), which prevents the effect of 'catastrophic' helicity quenching (e.g. Brandenburg and Subramanian, 2005). In our model, we don't include the helicity transport and consider the helicity quenching with a free parameter.

In this paper, we consider nonlinear behavior of the Parker's dynamo model (without magnetic helicity) and the Kleeorin-Ruzmaikin model (with magnetic helicity), and show that the latter can reproduce the qualitative behavior of the sunspot number variations during the solar cycles, including periodic and chaotic solutions for conditions of the solar convective zone. For simplicity, we use a "low-order model" approach (e.g. Weiss et al. 1984, Sokoloff and Nefyodov 2007), reducing the dynamo equations to a simple nonlinear dynamical system. In section 2, for consistency, we reproduce the formulations of the Parker's and Kleeorin-Ruzmaikin's models, and discuss conditions of linear stability and solar parameters. In section 3, we present the numerical solutions and compare these with observed properties of the sunspot number variations in solar cycles.

\section{Formulation}

\subsection{Parker's migratory dynamo}

In a kinematic approximation the dynamo problem can be described by the induction equation (Parker 1955)

$$
\frac{\partial \mathbf{B}}{\partial t}=\nabla \times(\mathbf{v} \times \mathbf{B})+\eta_{m} \nabla^{2} \mathbf{B}
$$

where $\mathbf{B}$ is the vector of magnetic field, $\mathbf{v}$ is the vector of fluid velocity, $\eta_{m}=1 /(\mu \sigma)$ is the molecular magnetic diffusivity. The magnetic field, $\mathbf{B}$, and the fluid velocity, $\mathbf{v}$, can be separated in two components representing mean and fluctuating (turbulent) parts, or $\mathbf{B}=\langle\mathbf{B}\rangle+\mathbf{b}$ and $\mathbf{v}=\langle\mathbf{v}\rangle+\mathbf{u}$. Here $\langle\mathbf{B}\rangle$ represents the averaged over longitude magnetic field, $\mathbf{b}$ is the fluctuating part of $\mathbf{B},\langle\mathbf{v}\rangle$ represents mean global-scale motions in the Sun, (such as the differential rotation), $\mathbf{u}$ is velocity of turbulent convective motions. Taking into account that the average of fluctuations is zero $(\langle\mathbf{b}\rangle=\mathbf{0}$ and $\langle\mathbf{u}\rangle=\mathbf{0})$ for the case of isotropic turbulence we obtain the following mean-field induction equation (e.g. Moffatt 1978)

$$
\frac{\partial\langle\mathbf{B}\rangle}{\partial t}=\nabla \times(\langle\mathbf{v}\rangle \times\langle\mathbf{B}\rangle+\alpha\langle\mathbf{B}\rangle-\eta \nabla \times\langle\mathbf{B}\rangle)
$$

where $\eta$ describes the total magnetic diffusion, which is the sum of the turbulent and molecular magnetic diffusivity, $\eta=\eta_{t}+\eta_{m}$ (usually $\eta_{m} \ll \eta_{t}$ ); parameter $\alpha$ is helicity. The first term of the equation describes 


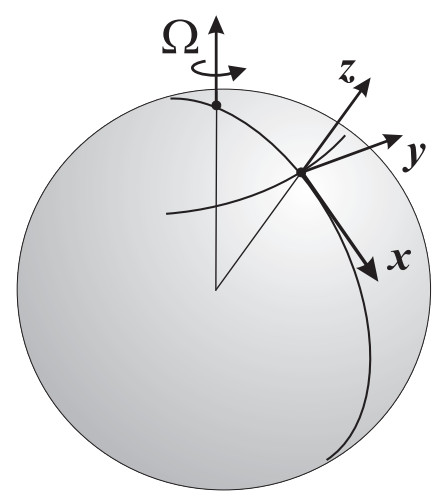

Figure 1. Local Cartesian reference coordinate system.

transport of magnetic field lines with fluid, the second term describes the $\alpha$-effect, and the last term determines diffusion and dissipation of the field.

For describing the average magnetic field, following Parker (1955), we choose a local coordinate system, $x y z$, where axis $z$ will represents the radial coordinate, axis $y$ is the azimuthal coordinate and axis $x$ coincides with colatitude (figure 1). Effects of sphericity are not included in this model. Hence, the vector of the mean field, $\langle\mathbf{B}\rangle$, can be represented as

$$
\langle\mathbf{B}\rangle=B_{y}(x, y) \mathbf{e}_{y}+\nabla \times\left[A(x, y) \mathbf{e}_{y}\right]
$$

where $B_{y}(x, y)$ is the toroidal component of magnetic field, $A(x, y)$ is the vector-potential of the poloidal field. Assuming that $\langle\mathbf{v}\rangle=v_{y}(x) \mathbf{e}_{u}$ (rotational component) we can write the dynamical system describing Parker's model of the $\alpha$-dynamo (Parker 1955) in the standard form:

$$
\begin{aligned}
& \frac{\partial A}{\partial t}=\alpha B+\eta \nabla^{2} A \\
& \frac{\partial B}{\partial t}=G \frac{\partial A}{\partial x}+\eta \nabla^{2} B
\end{aligned}
$$

where $G=\partial\left\langle v_{y}\right\rangle / \partial z$ is the rotational shear. Here for simplicity we omit the subscript of $B$.

Assuming that the coefficients are constants and seeking a solution of the model in the form $\left(A, B_{y}\right) \sim$ $\left(A_{0}, B_{0}\right) \exp [\mathrm{i}(k x-\omega t)]$, we find the well-known result that a pure periodic solution exists if

$$
D=\frac{\alpha G}{\eta^{2} k^{3}}=2
$$

where $D$ is the so-called "dynamo number". The solutions grow in time for $D>2$, and decay for $D<2$. As described in section 3, in the one-mode approximation the classical Parker's dynamo model even in nonlinear cases gives only non-chaotic oscillatory solutions, and, therefore, cannot explain the observed variations of the sunspot number in the solar cycles. For creating chaotic variations of the magnetic field in the lowmode approximation it is necessary to add to the Parker's model a third equation describing variations of the magnetic helicity and its interaction with the large-scale magnetic field (Kleeorin and Ruzmaikin 1982, Kleeorin et al. 1995). We note that the absence of chaotic solutions in the Parker's model with a simple algebraic quenching may be caused by the low-order truncation (Schmalz and Stix 1991), and that in a general PDE formulation chaotic solutions may appear (Covas et al. 1997, 1998).

\section{$2.2 \quad$ The Kleeorin-Ruzmaikin model}

In this section for clarity we reproduce the derivation of the equation for the magnetic helicity variations following Kleeorin and Ruzmaikin (1982). For this we consider helicity $\alpha$ as a variable consisting of two 
parts, hydrodynamic $\left(\alpha_{h}\right)$ and magnetic $\left(\alpha_{m}\right)$ (Pouquet et al. 1976, Kleeorin et al. 1995):

$$
\begin{gathered}
\alpha=\alpha_{h}+\alpha_{m}, \\
\alpha_{h}=-\tau\langle\mathbf{u} \cdot(\nabla \times \mathbf{u})\rangle / 3, \quad \alpha_{m}=\tau\langle\mathbf{b} \cdot(\nabla \times \mathbf{b})\rangle /(12 \pi \rho),
\end{gathered}
$$

where $\tau \sim l_{0}^{2} / \eta_{t}$ is the lifetime of turbulent eddies, $l_{0}$ is the characteristic scale of turbulent motions in the convective zone, and $\eta_{t}$ is the turbulent diffusion coefficient. It is convenient to define the influence of the magnetic helicity on magnetic field using spectral density $\chi(k)$ (Kleeorin and Ruzmaikin 1982)

$$
\bar{\chi} \equiv\langle\mathbf{a} \cdot \mathbf{b}\rangle=\int \chi(k) \mathrm{d} k
$$

where $\mathbf{a}$ is the fluctuating part of the vector-potential of magnetic field, $\mathbf{A}$, and $k$ is a wavevector.

To derive an equation for the averaged helicity density we multiply the basic induction equation written without the differential rotation term

$$
\frac{\partial \mathbf{B}}{\partial t}=\nabla \times\left(\mathbf{v} \times \mathbf{B}-\eta_{m} \nabla \times \mathbf{B}\right)
$$

by the fluctuating part of the vector potential, a; and also multiply the equation for the vector-potential

$$
\frac{\partial \mathbf{A}}{\partial t}=\mathbf{v} \times \mathbf{B}-\eta_{m} \nabla \times \nabla \times \mathbf{A}
$$

by the fluctuating part of magnetic field $\mathbf{b}$, and finally sum these equations. Then, using the averaging we obtain

$$
\begin{aligned}
\frac{\partial \bar{\chi}}{\partial t}=\left\langle\mathbf{a} \cdot \frac{\partial \mathbf{b}}{\partial t}+\mathbf{b} \cdot \frac{\partial \mathbf{a}}{\partial t}\right\rangle=\langle\mathbf{a} \cdot(\nabla \times(\mathbf{v} \times\langle\mathbf{B}\rangle))\rangle+\langle\mathbf{a} \cdot(\nabla \times(\mathbf{v} \times \mathbf{b}))\rangle+\langle\mathbf{b} \cdot(\mathbf{v} \times\langle\mathbf{B}\rangle)\rangle+ \\
\left.+\langle\mathbf{b} \cdot(\mathbf{v} \times \mathbf{b})\rangle-\eta_{m}(\langle\mathbf{a} \cdot(\nabla \times(\nabla \times \mathbf{b}))\rangle+\langle\mathbf{b} \cdot(\nabla \times \mathbf{b}))\rangle\right) .
\end{aligned}
$$

Taking into account that $\mathbf{b}=\nabla \times \mathbf{a}$, after some transformations we obtain the following expression for the helicity density

$$
\frac{\partial \bar{\chi}}{\partial t}=-2\langle[\mathbf{v} \times \mathbf{b}] \cdot\langle\mathbf{B}\rangle\rangle-2 \eta_{m}\langle\mathbf{b} \cdot \nabla \times \mathbf{b}\rangle .
$$

Two terms $\langle\triangle[\mathbf{a} \times[\mathbf{v} \times\langle\mathbf{B}\rangle]]\rangle$ and $\langle\triangle[\mathbf{a} \times[\mathbf{v} \times \mathbf{b}]]\rangle$, disappear as a result of averaging over the volume. Using the mean-field electrodynamics approximation and retain only the first two terms for the mean electric field (Moffatt 1978)

$$
\varepsilon \equiv\langle\mathbf{v} \times \mathbf{b}\rangle \cong \alpha\langle\mathbf{B}\rangle-\eta(\nabla \times\langle\mathbf{B}\rangle)
$$

we obtain

$$
\frac{\partial \bar{\chi}}{\partial t}=2\left(\eta\langle\mathbf{B}\rangle \cdot(\nabla \times\langle\mathbf{B}\rangle)-\alpha\langle\mathbf{B}\rangle^{2}-\eta_{m}\langle\mathbf{b} \cdot \nabla \times \mathbf{b}\rangle\right)
$$

Then we find a relationship between $\alpha_{m}$ and $\bar{\chi}$. For this we assume that in inertial range $\left(k_{0}, k_{1}\right)$ the last term of (15) can be written as

$$
\langle\mathbf{b} \cdot(\nabla \times \mathbf{b})\rangle=\int_{k_{0}}^{k_{1}} k^{2} \chi(k) \mathrm{d} k,
$$


where the helicity density spectrum, $\chi(k)$, can be described as (Kleeorin and Ruzmaikin 1982, Kleeorin et al. 1995, Zeldovich et al. 1983):

$$
\chi(k)=\bar{\chi} \frac{q-1}{k_{0}}\left(\frac{k_{0}}{k}\right)^{q}\left[1-\left(\frac{k_{0}}{k_{1}}\right)^{q-1}\right]^{-1} .
$$

Here $k_{0}^{-1}$ is an outer scale of a turbulence, $k_{1}^{-1}$ is the scale of the helicity spectrum cutoff. The value of parameter $q$ depends on the type of nonlinear interactions in the turbulent plasma. For example, $q=5 / 3$ describes the case of the Kolmogorov spectrum, and $q=3 / 2$ corresponds to turbulence of interacting Alfvén waves (Kraichnan's spectrum). In addition, factor $q$ was selected in such a way that coefficient $\chi$ coincides with the mean helicity. Consequently, the magnetic helicity given by (8) becomes

$$
\alpha_{m}=\frac{\tau}{12 \pi \rho}\langle\mathbf{b} \cdot(\nabla \times \mathbf{b})\rangle=\frac{1}{12 \pi \rho} \int_{k_{0}}^{k_{1}} k^{2} \tau_{*}(k) \chi(k) \mathrm{d} k
$$

where $\tau_{*}=2 \tau_{0}\left(k / k_{0}\right)^{1-q}, \tau_{0}$ is relaxation time, averaged over the energy spectrum.

Taking into account that $|\bar{\chi}|=|\langle\mathbf{a b}\rangle| \sim B^{2} / k_{0}$ and $\eta_{t}=\left(12 \tau_{0} k_{0}^{2}\right)^{-1}$, and substituting (17) into (18) we obtain

$$
\alpha_{m}=I \bar{\chi},
$$

where

$$
I=\frac{1}{18}\left(\frac{q-1}{2-q}\right) \frac{1}{4 \pi \rho \eta_{t}}\left(\frac{k_{*}^{4-2 q}-1}{1-1 / k_{*}^{q-1}}\right)
$$

and $k_{*}=k_{1} / k_{0}$.

Finally, multiplying equations (15) and (20) we obtain the expression for variations of the magnetic helicity in terms of the mean magnetic field:

$$
\frac{\partial \alpha_{m}}{\partial t}=\frac{Q}{2 \pi \rho}\left[\langle\mathbf{B}\rangle \cdot(\nabla \times\langle\mathbf{B}\rangle)-\frac{\alpha}{\eta}\langle\mathbf{B}\rangle^{2}\right]-\frac{\alpha_{m}}{T}
$$

where

$$
Q=\frac{1}{18}\left(\frac{q-1}{2-q}\right)\left(\frac{k_{*}^{4-2 q}-1}{1-1 / k_{*}^{q-1}}\right), \quad T=\frac{1}{2 \eta_{m} k_{0}^{2}}\left(\frac{3-q}{q-1}\right)\left(\frac{1-1 / k_{*}^{q-1}}{k_{*}^{3-q}-1}\right)
$$

Equation (21) is written for the case of an uniform turbulent diffusion $\left(\eta_{t}=\left(6 \tau_{0} k_{0}^{2}\right)^{-1}\right)$, and when the magnetic Reynolds number is large, $\eta \approx \eta_{t}$. In this model we do not include transport of magnetic field by flows.

For further analysis of the Kleeorin-Ruzmaikin model we transform equations (4)-(5) and (21) in a nonlinear dynamical system in non-dimensional variables. Following the approach of Weiss et al. (1984) we average the system of equations (4-5 and 21) in a vertical layer to eliminate $z$-dependence of $A$ and $B$ and consider a single Fourier mode propagating in the $x$-direction assuming $A=A(t) e^{\mathrm{i} k x}, B=B(t) e^{\mathrm{i} k x}$; then we get the following system of equations

$$
\begin{gathered}
\frac{\mathrm{d} A}{\mathrm{~d} t}=\alpha B-\eta k^{2} A, \quad \frac{\mathrm{d} B}{\mathrm{~d} t}=\mathrm{i} k G A-\eta k^{2} B, \\
\frac{\mathrm{d} \alpha_{m}}{\mathrm{~d} t}=-\frac{\alpha_{m}}{T}-\frac{Q}{2 \pi \rho}\left[-A B k^{2}+\frac{\alpha}{\eta}\left(B^{2}-k^{2} A^{2}\right)\right] .
\end{gathered}
$$


This transformation allows us to investigate more easily various nonlinear regimes, from periodic to chaotic, and obtain relationships of the basic properties, such as the cycle growth and decay times, duration and amplitude. However, we note that the formulation and the interpretation of solutions of the simplified system are not straightforward because it does not adequately describes nonlinear coupling of the spatial harmonics. Recently, Sokoloff and Nefyodov (2007) developed a self-consistent method of reducing the dynamo equations, (10) and (11), to a dynamical system. In particular, it follows from their study that the dominant modes of the toroidal field in the case of the solar dynamo are described by the harmonics, which are antisymmetric with respect to the equator (in accordance with the Hale law), $\sin (k x)$, where $x$ is colatitude, and the wavenumber, $k$, is even: $k=2,4, \ldots$ The first $k=2$ harmonic has the largest growth rate. We retain only this mode in our dynamical model. This nonlinear dynamical system is solved numerically by using standard Mathematica software for high-precision integration of potentially stiff problems.

For interpretation of solutions of the dynamical system in terms of the sunspot number properties we use the imaginary part of the toroidal component $B(t)$ because it gives the amplitude of the antisymmetric harmonics, and approximate the sunspot number, $W$, as $(\operatorname{Im} B)^{3 / 2}$, following Bracewell's suggestion. Of course, there might be different definitions of the relationship between characteristics of the magnetic field and the sunspot number parameter. We note that the solutions of the dynamical system are qualitatively similar for the different harmonics. Nevertheless, we choose the parameters, which correspond to the solar situation.

Making the following substitutions: $A=A_{0} \hat{A}, B=B_{0} \hat{B}, t=T_{0} \hat{t}, k=\hat{k} / r(r$ is a layer radius $)$, $T_{0}=1 /\left(k^{2} \eta\right)$ and $\alpha_{m}=\alpha_{0} \hat{\alpha}_{m}$, and taking into account that $A_{0}=B_{0} \eta k / G$ we obtain:

$$
\begin{gathered}
\frac{\mathrm{d} \hat{A}}{\mathrm{~d} \hat{t}}=\hat{D} \hat{B}-\hat{A}, \quad \frac{\mathrm{d} \hat{B}}{\mathrm{~d} \hat{t}}=\mathrm{i} \hat{A}-\hat{B}, \\
\frac{\mathrm{d} \hat{\alpha}_{m}}{\mathrm{~d} \hat{t}}=-\nu \hat{\alpha}_{m}+\left[\hat{A} \hat{B}-\hat{D}\left(\hat{B}^{2}-\lambda \hat{A}^{2}\right)\right],
\end{gathered}
$$

where $\hat{D}=D_{0} \hat{\alpha}$ and $\hat{\alpha}=\hat{\alpha}_{h}+\hat{\alpha}_{m}$ are the non-dimensional dynamo number and helicity, $D_{0}=\alpha_{0} G r^{3} / \eta^{2}$, $\alpha_{0}=2 Q k v_{A}^{2} / G, v_{A}$ is the Alfvén speed, $\nu=T_{0} / T$ (here we assume that $k_{0}$ is close to $k_{1}$ then $T \sim$ $1 /\left(2 k_{0}^{2} \eta_{m}\right)$, (Kleeorin and Ruzmaikin 1982)) and $\lambda=\left(k^{2} \eta / G\right)^{2}=\mathrm{Rm}^{-2}, k$ is a characteristic wavelength, $\mathrm{Rm}$ is the magnetic Reynolds number. In the next section we discuss the ratio of parameters when the variations become autonomous.

\section{$2.3 \quad$ Linear stability}

First, we simplify the third equation of system (24) by neglecting the last term, because $\lambda$ is very small for large magnetic Reynolds numbers. Note, that hereafter we omit the hat symbol for all non-dimensional variables. Hence (24) becomes

$$
\begin{aligned}
\frac{\mathrm{d} A}{\mathrm{~d} t} & =D B-A, \quad \frac{\mathrm{d} B}{\mathrm{~d} t}=\mathrm{i} A-B, \\
\frac{\mathrm{d} \alpha_{m}}{\mathrm{~d} t} & =-\nu \alpha_{m}+\left[A B-D B^{2}\right] .
\end{aligned}
$$

Using a linearization procedure, $A=A^{\prime}+A^{0}, B=B^{\prime}+B^{0}$ and $\alpha=\alpha_{m}^{\prime}+\alpha_{h}$, and taking into the account that the dynamo number is $D=D_{0} \alpha$, we obtain the following equations

$$
\begin{aligned}
\frac{\mathrm{d} A^{\prime}}{\mathrm{d} t} & =D_{0} \alpha_{h} B^{0}-A^{\prime}, \quad \frac{\mathrm{d} B^{\prime}}{\mathrm{d} t}=\mathrm{i} A^{\prime}-B^{\prime}, \\
\frac{\mathrm{d} \alpha_{m}^{\prime}}{\mathrm{d} t} & =-\nu \alpha_{m}^{\prime}+\left[A^{0} B^{\prime}+A^{\prime} B^{0}-2 D_{0} \alpha_{h} B^{\prime} B^{0}-2 D_{0} \alpha_{m}^{\prime} B_{0}^{2}\right] .
\end{aligned}
$$


Then, considering solutions in the form $\sim e^{-i \omega t}$ we obtain the condition of linear stability: $\left|D_{0} \alpha_{h}\right|>2$, in the approximation of $B^{0}=0$ and $A^{0}=0$. Thus, in the case of $\left|D_{0} \alpha_{h}\right|<2$ the magnetic field does not grow. For $\left|D_{0} \alpha_{h}\right|=2$ we have a periodical solution like in the Parker's model. The linear theory also provides the direction of the migration of the dynamo waves with latitude. These waves travel from higher to lower latitudes if $D$ is negative, and in the opposite direction for positive $D$. Thus, to make the model consistent with the butterfly diagram we consider the case of negative $D$.

\subsection{Solar parameters}

In order to estimate the range of parameters of the Kleeorin-Ruzmaikin model, and for modeling the solar cycle we used the standard model of the interior structure of the Sun for the top, bottom and middle areas of the convective zone (Table 1). The key parameter of the model is the dynamo number $D=D_{0} \alpha$, because its magnitude determines behavior of the magnetic field. We remind that according to the condition of linear stability, $D_{0} \alpha_{h}$ should be greater than 2. Taking into account that $D_{0}=\alpha_{0} G r^{3} / \eta^{2}$, we assume that $\eta \approx \eta_{t}, G \sim\left\langle v_{y}\right\rangle / r$, where $\left\langle v_{y}\right\rangle$ is a typical rotational velocity, $r$ is the radius of convective layers. Parameter $\lambda$ determines the influence of vector-potential $A$ on variations of magnetic helicity $\alpha_{m}$. From our estimates it follows that for the solar conditions $\lambda \leq 10^{-4}$. Consequently, we can neglect the term with $\lambda$. The last non-dimensional parameter, $\nu$, included in equations (24) describes the ratio of two characteristic times, $T_{0}$ and $T$. Parameter $T_{0}=1 /\left(k^{2} \eta\right)$ is estimated using the values of turbulent diffusivity $\eta_{t}=1.5 H_{p} V_{\text {conv }}$ from the mixing-length model, where $H_{p}$ is the pressure scale height, $V_{\text {conv }}$ is the convective velocity. In the absence of helicity fluxes the value of the damping parameter $\nu$ is small. However, in reality the helicity fluxes increase the dissipation rate. This can be modeled as a damping term, which increases the effective value of $\nu$ (Blackman and Brandenburg 2003). Because of this the value of $\nu$ is to some extent uncertain.

\section{Numerical calculations}

\subsection{Periodic solutions}

According to the model of Parker (equations 4 and 5), the pure harmonic variations of the magnetic field occur for the dynamo-number $D=2$. It is also known, that for $|D|>2$ we have solutions with increasing amplitude. Following a standard procedure, we included a nonlinearity (alpha-quenching) as $\alpha_{h} /\left(1+\xi B^{2}\right)$ (Ivanova and Ruzmaikin 1977, Brandenburg and Subramanian 2005), where $\xi$ is a quenching parameter, which limits the growth of the magnetic field amplitude. Examples of nonlinear solutions for the Parker's model are shown in figure 2. However, our numerical calculation showed that in the nonlinear regime the variations of the toroidal field (figure 2a) and the sunspot number (figure 2b) are also periodic and similar to the classical case of the linear harmonic solution for $|D|=2$. For calculations of the model sunspot number we used Bracewell's suggestion (private communication) of the connection between the toroidal field strength, $B$, and the sunspot number, $W$, in the form of the three-halfs law: $W \sim B^{3 / 2}$.

We note that the model shows a larger amplitude of variations for the poloidal component than for the toroidal field, and that there is a phase shift between them. However, comparing of the observed profiles

Table 1. Parameters of the standard model for the different parts of the solar convective zone.

\begin{tabular}{lccc}
\hline Parameter & bottom & middle & top* \\
\hline radius $(r), \mathrm{cm}$ & $5 \times 10^{10}$ & $6 \times 10^{10}$ & $7 \times 10^{10}$ \\
density $(\rho), \mathrm{g} / \mathrm{cm}^{3}$ & $2 \times 10^{-1}$ & $4 \times 10^{-2}$ & $2 \times 10^{-3}$ \\
turbulent diffusivity $\left(\eta_{t}\right), \mathrm{cm}^{2} / \mathrm{s}$ & $2 \times 10^{13}$ & $3 \times 10^{13}$ & $1 \times 10^{13}$ \\
radial velocity gradient $(G)^{* *}, \mathrm{~s}^{-1}$ & $5 \times 10^{-6}$ & $2 \times 10^{-6}$ & $-2 \times 10^{-6}$ \\
\hline
\end{tabular}

* Supergranulation layer.

** Calculated from the helioseismology inversion results of Schou et al. (1998) 

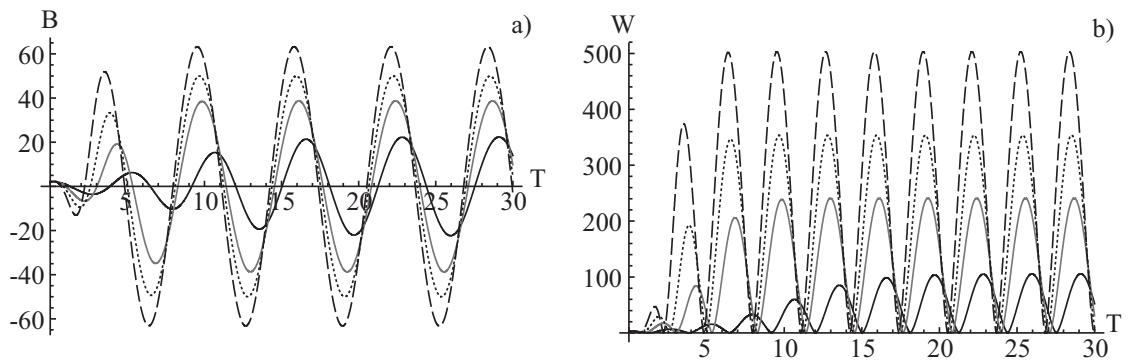

Figure 2. Solutions (in non-dimensional units) for the toroidal magnetic field (a) and the sunspot number (b) in case the Parker's dynamo model for different values of the dynamo number, $D$, and quenching parameter $\xi=10^{-3}: D=3$ (solid black curve), $D=5$ (grey curve), $D=7$ (dotted curve) and $D=10$ (dashed curve).

of the solar cycles (figure 9) with the solutions for the Parker model (figure 2b), we see that, in general, the model solutions have a sinusoidal character and do not describe the basic properties of the sunspot profiles with rapid growth and slow decay.

For the Kleeorin-Ruzmaikin model, given by equations (24), the linear instability condition is also $|D| \equiv\left|\alpha_{h} D_{0}\right|>2$. However, in this case the profile of the periodic solutions is not sinusoidal, and depends on the initial conditions, $A_{0}$ and $B_{0}$. For higher initial values the amplitude of the nonlinear oscillations in the stationary state is higher. However, the shapes of the oscillation profiles are similar.

Figure 3 illustrates solutions for the model of Kleeorin-Ruzmaikin, and the corresponding variations of the sunspot number for different initial conditions. As mentioned, changes of initial values for magnetic field components $A_{0}$ and $B_{0}$ leads to very similar profiles. In high amplitude cases, dual peaks may appear in the variations of the vector potential, $A$, of the poloidal field. The evolution of the magnetic helicity represents a relatively smooth growth followed by a sharp decay. The helicity has maxima when the toroidal field is zero. In these calculations the value of parameter $\nu$, which describes damping rate of magnetic helicity and depends on the turbulence spectrum and the dissipation though helicity fluxes, is of the order of unity. Finally, the variations of the sunspot number, $W$, with the amplitude increase are characterized by higher peaks and shorter rising times (figure 3d). Note that in the sunspot number profile we can recognize the well-known general properties of the sunspot number profile with the rapid growth at the beginning of a cycle and a slow decrease after the maximum.

With the increase of $\left|\alpha_{h} D_{0}\right|\left(\left|\alpha_{h} D_{0}\right|>2\right)$ the profile of magnetic field variations continue to deform and can become unstable with very steep variations of the magnetic field. The solution can be stable again if we enhance the back reaction by increasing the quenching parameter. We use the following quenching
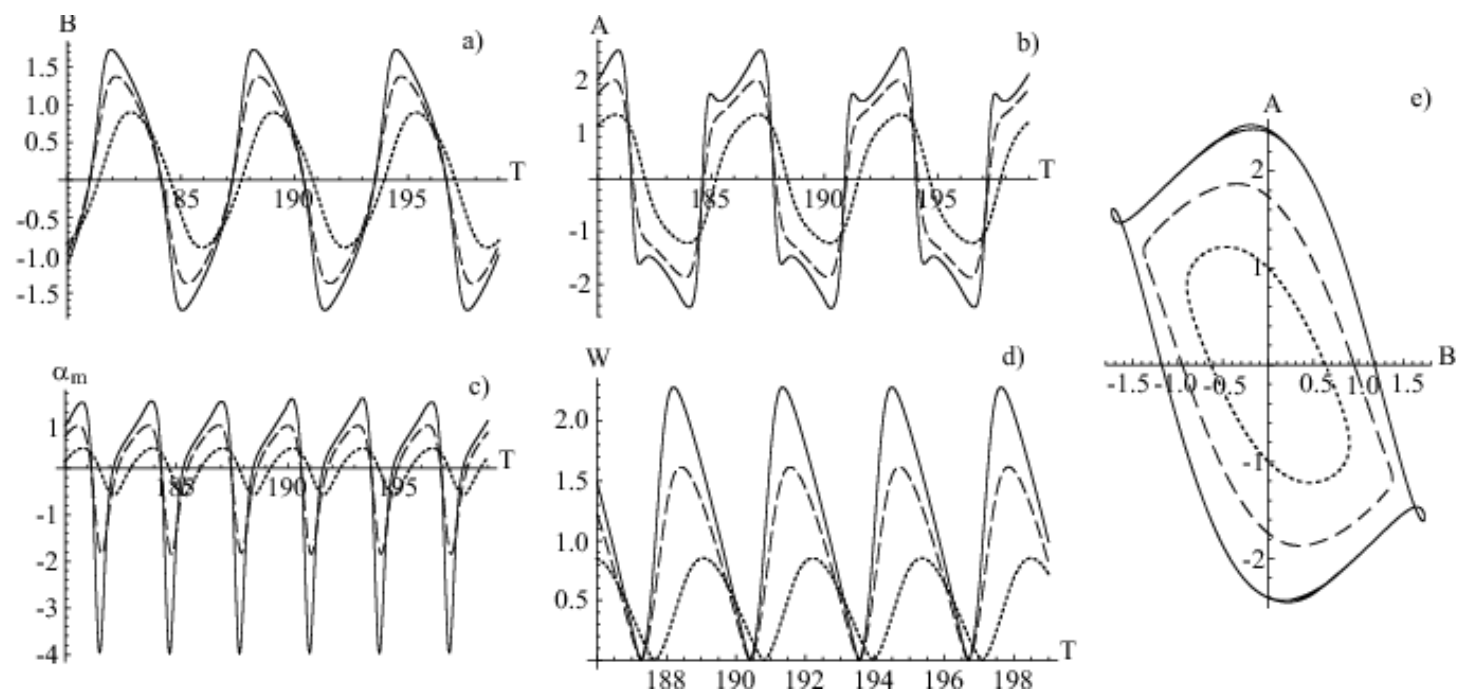

Figure 3. Variations of the magnetic field for the middle convective zone $\alpha_{h} D_{0}=-2: \nu=1.28, \alpha_{h}=2.439, D_{0}=-0.82$ for different initial conditions: $B_{0}=4 \mathrm{i}, A_{0}=-0.01 \mathrm{i}$ (dotted curve), $B_{0}=4 \mathrm{i}, A_{0}=-\mathrm{i}$ (dashed curve) and $B_{0}=1+4 \mathrm{i}, A_{0}=-\mathrm{i}$ (black curve): a) toroidal component, $B ; \mathrm{b}$ ) vector-potential, $A$, of the poloidal magnetic field; c) magnetic helicity variations; d) evolution of the model sunspot number; e) phase portrait of the magnetic field components. 

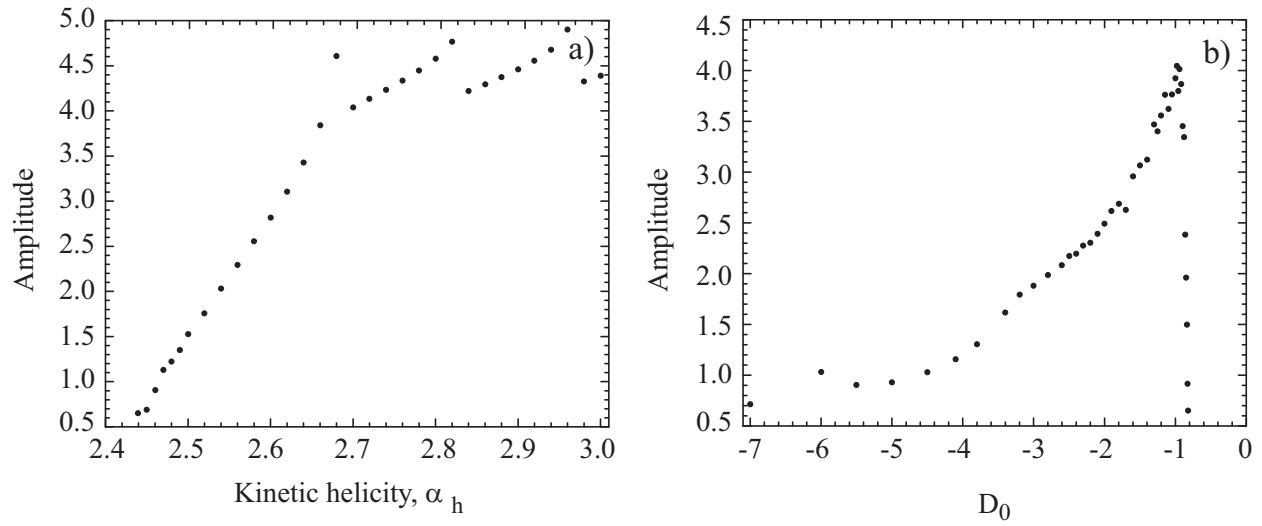

Figure 4. Dependence of the amplitude of model sunspot number $W$ on a) kinetic helicity $\alpha_{h}$ at fixed dynamo number $D_{0}=-0.82$; b) dynamo number $D_{0}$ at fixed $\alpha_{h}=2.44$.

formula for the kinetic part of helicity, $\alpha_{h}$ ( Kleeorin et al. 1995)

$$
\alpha=\frac{\alpha_{h}}{1+\xi B^{2}}+\alpha_{m}
$$

Thus we always have a possibility for selecting $\xi$ to obtain periodic nonlinear solutions.

As mentioned earlier, the change in values of the dynamo number and the kinetic helicity leads to a change in the amplitude of the magnetic field variations (figure 4). With the increase of the kinetic helicity, $\alpha_{h}$, above the linear stability level at a fixed dynamo number (in this example, $D_{0}=-0.82$ ) the amplitude rapidly grows (figure 4a). However, for a fixed helicity value (e.g. $\alpha_{h}=2.44$ ) and increasing value of dynamo number $D_{0}$ the amplitude rapidly grows and then sharply declines (figure $4 \mathrm{~b}$ ). For other values of $D_{0}$ and $\alpha_{h}$, satisfying the linear instability condition, the solutions are qualitatively similar.

Figure 5 shows the relationships between of the amplitude of the sunspot number parameter, the growth and decay times and the cycle duration, for different values of $\alpha_{h}$ and $D_{0}$. These characteristic times were determined from the points of minima and maxima of the model sunspot number, $W$. The crosses represent the amplitude of the periodic field variations for $D_{0}=-0.82$ and different values of kinetic helicity, $\alpha_{h}$. The circles correspond to the case of constant $\alpha_{h}=2.44$ and different values of dynamo number $D_{0}$. In the first case, the relationship between the cycle amplitude and the growth time is well-defined and monotonic. However, in the case of a fixed $\alpha_{h}$ and varying $D_{0}$ (circles) the amplitude initially, at small $D_{0}$, follows the same sequence as in the variable $\alpha_{h}$ case, but then at higher values of $\left|D_{0}\right|$ (shown by bigger circles) the amplitude decreases. The decay time (figure 5b) is longer for higher amplitude cycles. The relationship between the amplitude and the cycle duration (figure 5c) is more complex. In the case
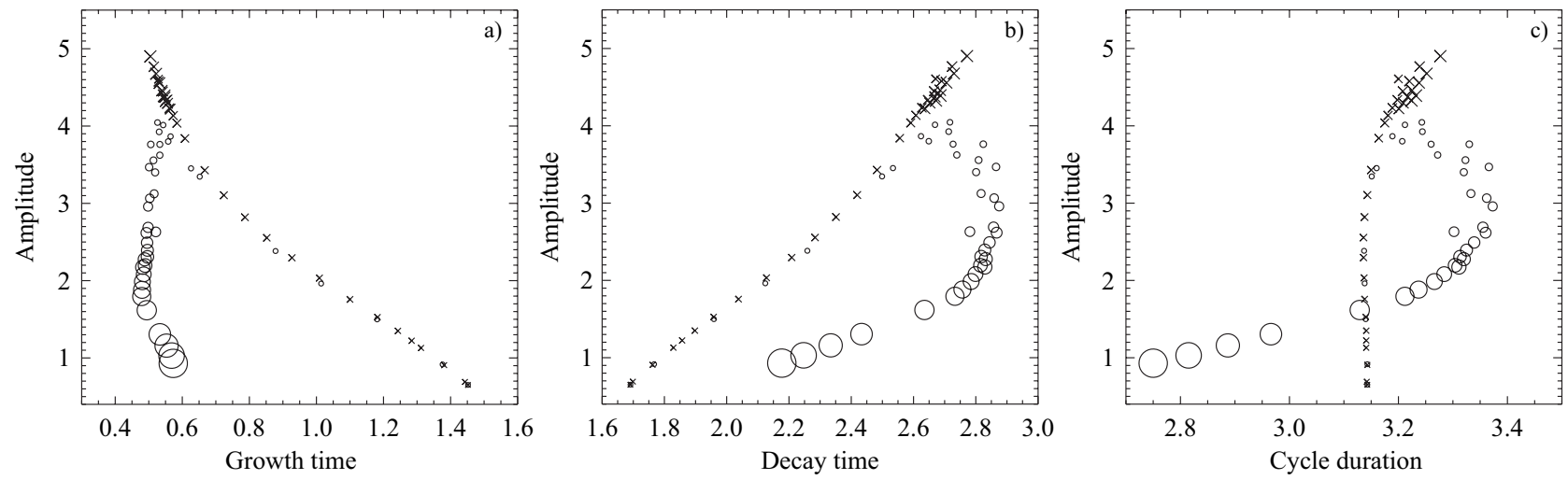

Figure 5. Relationships between the model sunspot number amplitude, $W$ and a) the cycle growth time, b) the decay time, and c) the cycle duration. The circles show a sequence for a fixed value of the kinetic helicity, $\alpha_{h}=2.44$ and the dynamo number varying from -7 to -0.82 . The crosses show the case of fixed $D_{0}=-0.82$ and varying $\alpha_{h}$, from 2.44 to 3 . The size of the crosses and circles is proportional to the corresponding values of $D_{0}$ and $\alpha_{h}$. Other model parameters correspond to the middle convective zone: $\nu=1.28, \lambda=1.23 \times 10^{-6} ;$ and the initial conditions are $A_{0}=-\mathrm{i}, B_{0}=\mathrm{i}, \alpha_{m}^{0}=0$. 
of fixed $D_{0}$ the amplitude and the cycle duration increase with the dynamo number, but initially at lower $\alpha_{h}$, just above the stability value, the amplitude rapidly grows but the duration does not change much. With further increase of $\alpha_{h}$ the amplitude grows slowly, but the cycles become longer. In the case of the varying dynamo number (circles) the relationship is multivalued, that is the cycles of the same duration may have different amplitudes.

\subsection{Chaotic solutions}

The transition of the periodic to chaotic solutions occurs when the dynamo number, $\left|\alpha_{h} D_{0}\right|$, increases. In the transition regime the cycle amplitude becomes modulated: it slowly increases with time, and then suddenly and very sharply declines, and then start growing again (figure 6a). Some examples of the transition to the chaotic regime for the sunspot number parameter and the development of chaotic behavior are shown in figure 6 for a fixed $D_{0}=-0.82$ and increasing values of the kinetic helicity, $\alpha_{h}$.

At small deviation from the periodic regime the slow rise of amplitude is followed by sharp recession. Nevertheless, each subsequent increase in the amplitude is almost identical to the previous one, or in other words, we have in this case, a modulated periodic solution (figures $6 \mathrm{a}, \mathrm{b}$ ). Also, we note that the shape of the profile for the each short cycle is still the same as in the case of the periodic solutions with the rapid growth and slow decline. With further increase of the kinetic helicity, the general behavior with growing cycle amplitudes and subsequent recessions continues, but the variations of amplitude become increasingly more chaotic (figures $6 \mathrm{c}, \mathrm{d}$ ). The amplitude of the solutions is controlled by the quenching parameter, $\xi$, which was chosen in figure 6 to obtain the solutions with amplitudes of the same order of magnitude.

In the case of significant deviations from the condition of linear stability the solutions become chaotic for all variables of the dynamical system. Figure 7 shows an example of chaotic variations for the magnetic field components, the magnetic helicity and the sunspot number parameter. In the chaotic solutions, the peaks of the toroidal magnetic field, $B$ (figure 7a) strongly correlate with the peaks of the vector-potential, $A$, and the magnetic helicity, $\alpha_{m}$, (figures $7 \mathrm{~b}, \mathrm{c}$ ). The growth of the toroidal field also leads to strengthening of the poloidal field and strong fluctuations of the magnetic helicity. The comparison of the variations for the toroidal component and vector-potential on the phase portrait (figure 7e) shows behavior resembling a strange attractor, when the trajectory converges to a steady state, then deviates from it, and returns again.

The peaks ("cycles") of the chaotic solutions still reproduce the typical profiles of the sunspot number
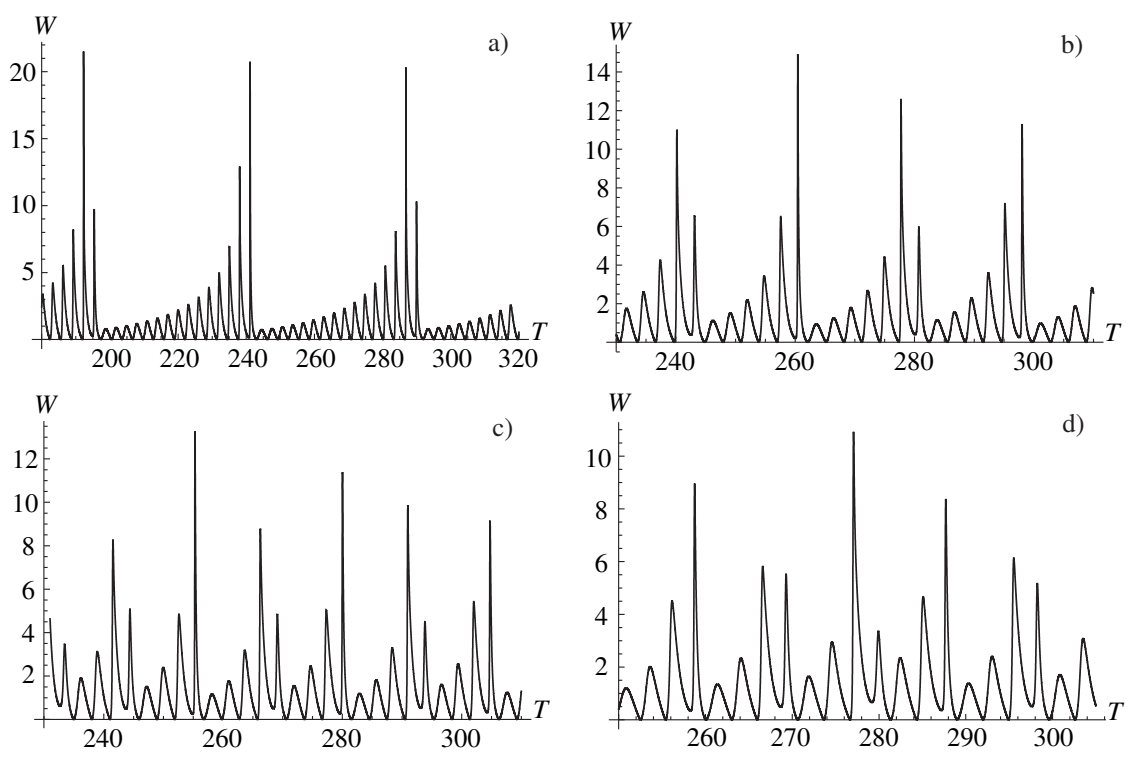

Figure 6. Variations of the model sunspot number, $W$, illustrating the transition from the periodic to chaotic solutions for conditions of the middle convective zone, dynamo number $D_{0}=-0.82, \nu=1.28, \lambda=1.23 \times 10^{-6}$ and different values of the kinetic helicity $\alpha_{h}$ $\left(D_{0} \alpha_{h}\right)$ : a) $\alpha_{h}=2.58, \xi=10^{-3}(-2.116)$, b) $\alpha_{h}=2.8, \xi=10^{-3}(-2.296)$, c) $\alpha_{h}=3, \xi=1.3 \times 10^{-3}(-2.46)$, and d) $\alpha_{h}=3.2$, $\xi=3.9 \times 10^{-3}(-2.624)$. 

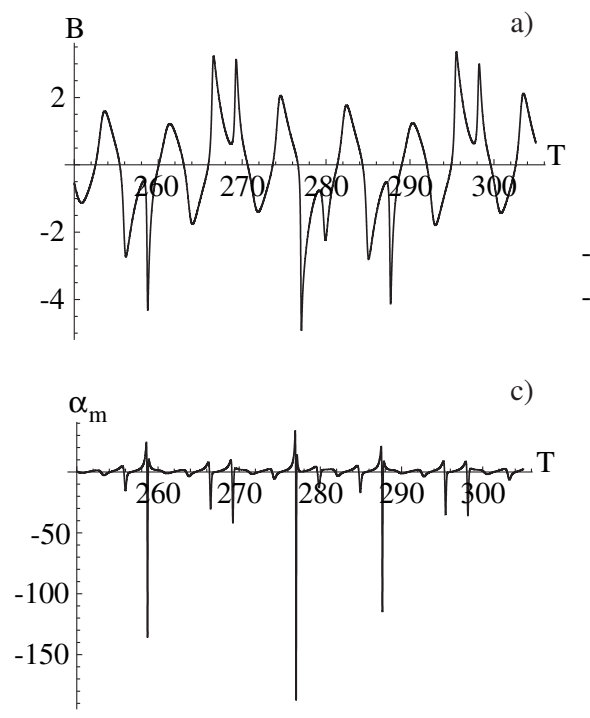

b)

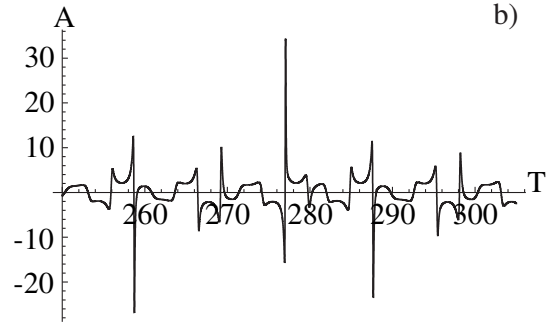

d)

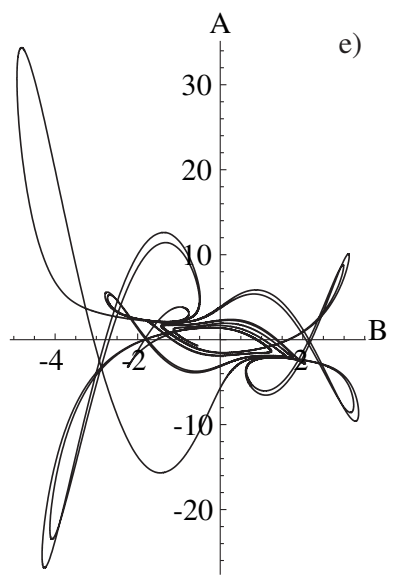

Figure 7. Example of the chaotic solution for the middle convective zone $\left(\nu=1.28, \lambda=1.23 \times 10^{-6}, D_{0}=-0.82, \alpha_{h}=3.2\right.$, $\left.\xi=3.9 \times 10^{-3}\right)$ : a) toroidal field $B, \mathrm{~b}$ ) vector-potential $A, \mathrm{c}$ ) magnetic helicity $\left.\alpha_{m}, \mathrm{~d}\right)$ model sunspot number $W$ and e) the phase portrait of the magnetic components.

variations described for the periodic solutions. However, the duration and amplitude of the cycles vary. For comparison in the figure 8 we show the properties of the different cycles of three chaotic solutions for $D_{0}=-0.82$ and: $\alpha_{h}=2.8$ (black circles), $\alpha_{h}=3$ (empty circles) and $\alpha_{h}=3.2$ (stars). In particular, we can see that the growth time is shorter for stronger cycle (figure 8a). The decay time, in general, is also longer for stronger cycle (figure 8b). However, the relationship between the cycle duration and its amplitude is again not very certain (figure 8c).

It is interesting to note that the similar relationships between the amplitude and the characteristic times, which we found for both periodic and chaotic solutions of the Kleeorin-Ruzmaikin model, occur for the real solar cycles (figures 9 and 10). For comparing the properties of the simulated variations of the sunspot number and the solar cycles we used the monthly data of the sunspot number (figure 9) for the period of 1750 - 2007 from National Geophysical Data Center (ftp://ftp.ngdc.noaa.gov/stp/solar_data/sunspot_numbers/monthly.plt). The growth and decay times were measured from the sunspot minimum to the maximum and from the maximum to the next minimum, using the same procedure as for the model. The results shown in figure 10 resemble the theoretical relationships of figures 5 and 8. In particular, the growth time is shorter for stronger cycles. The decay time and the duration do not show a clear correlation with the amplitude.
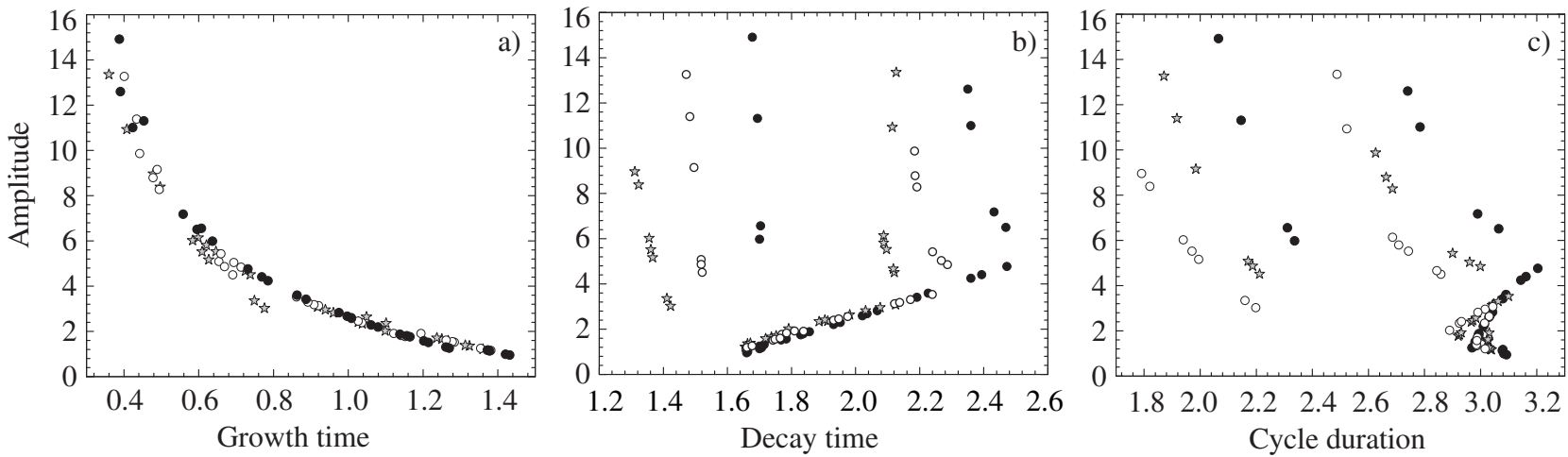

Figure 8. Relationships between the amplitude of the model sunspot number and a) the growth time, b) decay time and c) the cycle duration, for $D_{0}=-0.82$ and different values of the kinetic helicity: $\alpha_{h}=2.8$ (black circles), $\alpha_{h}=3$ (empty circles), $\alpha_{h}=3.2$ (stars). The time scales are non-dimensional (in units $T_{0}=1 /\left(k^{2} \eta\right)$ ). 


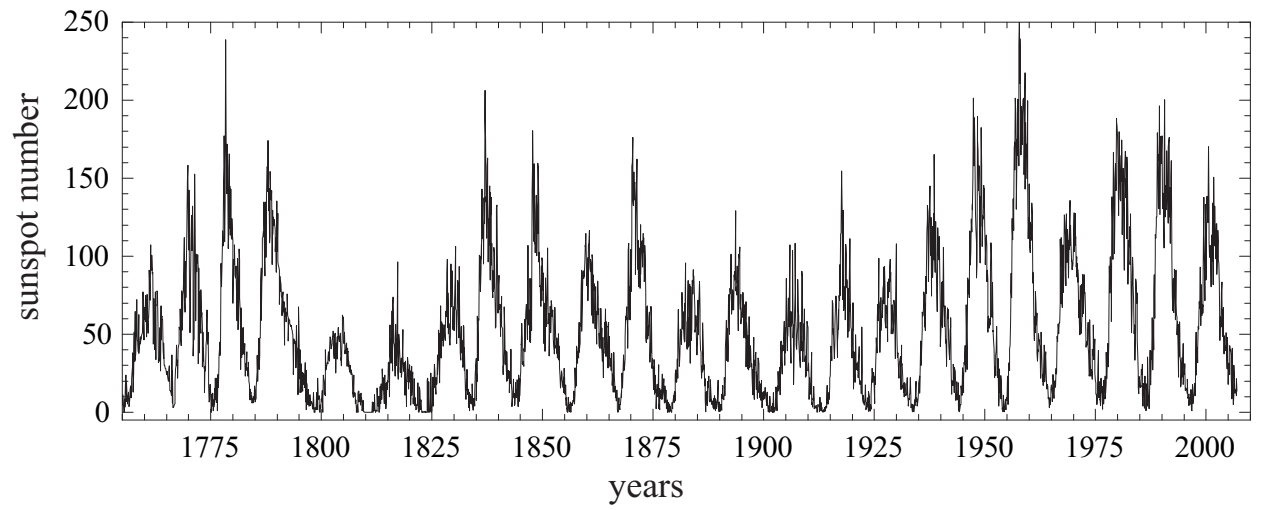

Figure 9. Observed monthly sunspot number series for 1755 - 2007 yrs. from National Geophysical Data Center.
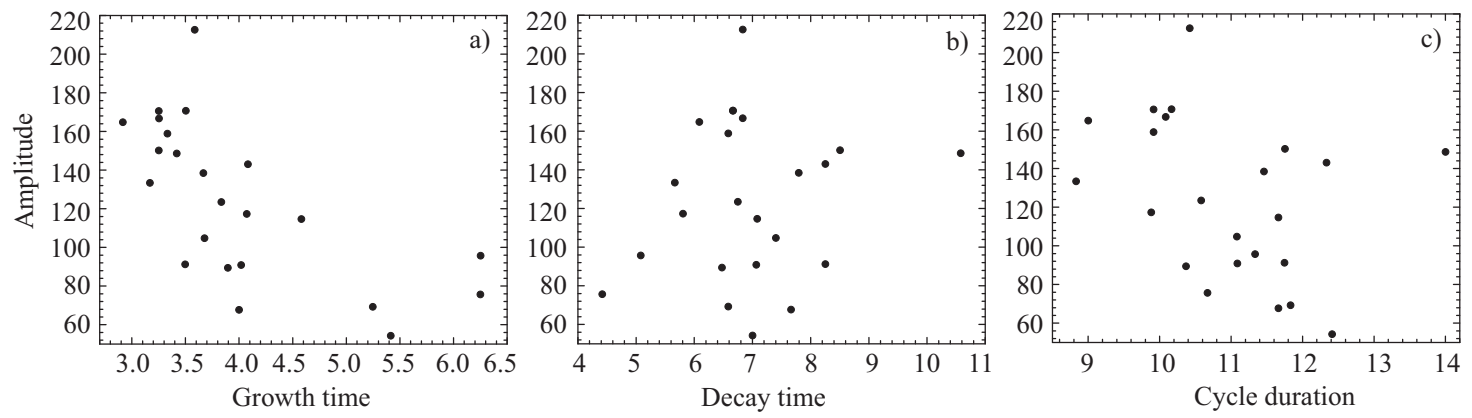

Figure 10. Properties of the solar cycles of 1755 - 2007: the relationships between the amplitude of the sunspot number and a) the cycle growth time, b) the cycle decay time and c) the cycle duration. The time scales are in years.

\section{Discussion}

We have presented a numerical analysis of simple dynamical models describing the nonlinear behavior of two dynamo models, the classical Parker's dynamo model with the standard $\alpha$-quenching and the model of Kleeorin and Ruzmaikin (1982), which takes into account effects of turbulent magnetic helicity. The models are analyzed in the low-mode approximation with the goal of representing variations of a sunspot number parameter during the solar cycles. We found that the Parker's model does not reproduce the typical behavior of the sunspot number with a fast growth and slow decay or obtain a chaotic solution in the low-mode approximation, even in strong nonlinear regimes. The analysis of the Kleeorin-Ruzmaikin model showed the existence of nonlinear periodic and chaotic solutions for conditions of the solar convective zone. For this model we obtained the profiles of the sunspot number variations, which qualitatively reproduce the typical profile of the solar cycles.

It is interesting that the properties of the simulated cycles demonstrate good qualitative agreements with the properties of the observed solar cycles. In particular, the relationship between the cycle amplitude and the growth time looks similar. Of course, such simplified models cannot pretend to provide a realistic description of the solar cycles. More complicated models such as the models with flux transport (e.g. Dikpati and Gilman 2006) should be considered for more detailed description of the solar magnetic field evolution.

Nevertheless, these results encourage further theoretical and observational investigations of the dynamo model with the turbulent magnetic helicity. We also hope that through the use of a simple dynamical model reproducing the basic properties of the solar cycle it will become possible to apply the data assimilation methods for predicting the solar cycles (e.g. Kitiashvili 2008). 


\section{REFERENCES}

Blackman, E.G. and Brandenburg, A., Doubly helical coronal ejections from dynamos and their role in sustaining the solar cycle Astrophys. J., 2003, 584, L99-L102.

Bracewell, R.N., The sunspots number series. Nature, 1953, 171, 649-650.

Bracewell, R.N., Three-halves law in sunspot cycle shape. Mon.Not.R.Astr.Soc, 1988, 230, 535-550.

Brandenburg, A. and Subramanian, K., Astrophysical magnetic fields and nonlinear dynamo theory. Physics Reports, 2005, 417, 1-209.

Charbonneau, P., A Maunder minimum scenario based on cross-hemispheric coupling and intermittency. Solar Physics, 2005, 229, 345-358.

Covas, E., Tavakol, R., Tworkowski, A. and Brandenburg, A., Axisymmetric mean field dynamos with dynamic and algebraic $\alpha$-quenchings. Astron. Astrophys., 1998, 329, 350-360.

Covas, E., Tworkowski, A., Brandenburg, A. and Tavakol, R., Dynamos with different formulations of a dynamic $\alpha$-effect. Astron. Astrophys., 1997, 317, 610-617.

Dikpati, M. and Gilman, P.A., Simulating and predicting solar cycles using a flux-transport dynamo. Astrophys. J., 2006, 649, 498-514.

Hale, G.E., Ellerman, F., Nicholson, S.B. and Joy, A.H., The magnetic polarity of Sun-spots. Astrophys. J., 1919, 49, 153-178.

Ivanova, T.S. and Ruzmaikin, A.A., A nonlinear magnetohydrodynamic model of the solar dynamo. Sov. Astron., 1977, 21, 479-485.

Kitiashvili, I., Application of data assimilation methods to non-linear solar dynamo models. ASP Conf. Series, 2008, 383, 255-263.

Kleeorin, N., Kuzanyan, K., Moss, D., Rogachevskii, I., Sokoloff, D. and Zhang, H., Magnetic helicity evolution during the solar activity cycle: observations and dynamo theory. Astron. Astrophys., 2003, 409, 1097-1105.

Kleeorin, N., Moss, D., Rogachevskii, I. and Sokoloff, D., Helicity balance and steady-state strength of the dynamo generated galactic magnetic field. Astron. Astrophys., 2000, 361, L5-L8.

Kleeorin, N., Rogachevskii, I. and Ruzmaikin, A., Magnitude of the dynamo-generated magnetic field in solar-type convective zones. Astron. Astrophys., 1995, 297, 159-167.

Kleeorin, N. and Rogachevskii, I., New mechanism of generation of large-scale magnetic field in a sheared turbulent plasma. Planetary and Space Science, 2007, 55, 2315-2318.

Kleeorin, N.I. and Ruzmaikin, A.A., Dynamics of the mean turbulent helicity in magnetic field. Magnetohydrodynamics, 1982, 18, 116-122.

Krause, F. and Rädler, K.-H., Mean-field magnetohydrodynamics and dynamo theory. 1980 (Pergamon Press, Oxford).

Moffatt, H.K., Magnetic field generation in electrically conducting fluids. 1978 (New York: Cambridge Univ. Press).

Parker, E.N., Hydromagnetic dynamo models. Astrophys. J., 1955, 122, 293-314.

Pouquet, A., Frisch, U. and Léorat, J., Strong MHD helical turbulence and the nonlinear dynamo effect. J. Fluid Mech., 1976, 77, 321-354.

Schou, J. and 23 colleagues, Helioseismic studies of differential rotation in the solar envelope by the solar oscillations investigation using the Michelson Doppler Imager. Astrophys. J., 1998, 505, 390-417.

Schmalz, S. and Stix, M., An $\alpha \Omega$ dynamo with order and chaos. Astron. Astrophys., 1991, 245, 654-661.

Schwabe, H., Sonnenbeobachtungen im Jahre 1843. Astronomische Nachrichten, 1844, 21(495), 233-236.

Sokoloff, D., Astrophysical dynamos and magnetic helicity conservation. Plasma Phys. Control. Fusion, 2007, 49, B447-B452.

Sokoloff, D., Zhang, H., Kuzanyan, K.M. and Tomin, D., Magnetic and current helicities in solar dynamos. Advances in Space Research, 2007, 39, 1670-1673.

Sokoloff, D.D. and Nefyodov, S.N., Low-mode approximation for stellar dynamos. Vychislitelinye methody i programmirovanie, 2007, 8, 195-204, (in Russian),(www.srcc.msu.ru).

Sur, S., Shukurov, A. and Subramanian, K., Galactic dynamos supported by magnetic helicity fluxes. Mon.Not.R.Astr.Soc, 2007, 377, 874-882. 
Zhang, H., Sokoloff, D., Rogachevskii, I., Moss, D., Lamburt, V., Kuzanyan, K. and Kleeorin, N., The radial distribution of magnetic helicity in the solar convective zone: observations and dynamo theory. Mon.Not.R.Astr.Soc, 2006, 365, 276-286.

Zeldovich, Ya.B., Ruzmaikin, A.A. and Sokoloff, D.D., Magnetic fields in astrophysics. 1982 (New York: Gordon and Breach Science Publishers).

Weiss, N.O., Cattaneo, F. and Jones C.A., Periodic and aperiodic dynamo waves. Geophys. Astrophys. Fluid Dynamics, 1984, 30, 305-341. 